\title{
Total resection in a giant left frontal arteriovenous malformation, grade V Spetzler-Martin - Case report
}

\author{
Aurelia Mihaela Sandu ${ }^{1}$, Mircea Radu Gorgan ${ }^{2}$ \\ ${ }^{1} \mathrm{PhD}$ Student in Neurosurgery, "Carol Davila" University of Medicine and \\ Pharmacy Bucharest, Faculty of Medicine, Department of Neurosurgery \\ Clinic of Neurosurgery, Fourth Department of Neurosurgery, Emergency Clinical \\ Hospital Bagdasar-Arseni, Bucharest \\ 2"Carol Davila" University of Medicine and Pharmacy Bucharest, Faculty of \\ Medicine, Department of Neurosurgery \\ Head of Clinic of Neurosurgery, Emergency Clinical Hospital Bagdasar-Arseni, \\ Bucharest
}

\begin{abstract}
Background: Giant arteriovenous malformations (AVMs) are congenital lesions, with nidus sizing more than $6 \mathrm{~cm}$. According to Spetzler-Martin scale, grade V AVMs have a nidus larger than $6 \mathrm{~cm}$ in diameter, profound venous drainage and are located in eloquent areas.

Method: We report a case of a 39 years old woman, with giant left frontal AVM, grade V Spetzler-Martin, who was admitted for generalized seizures, with onset 32 years ago, refractory to full dose antiepileptic polytherapy, which severely impaired the patient's quality of life.

Results: The patient underwent surgery and we performed total resection of the AVM. We emphasize on surgical technique, intraoperative difficulties and outcome.

Conclusions: Surgery is the therapy of choice in AVMs, because it provides cure of the lesion, and is the only treatment capable of preventing hemorrhage and controlling seizures. Management in grade V AVMs is challenging, because of their large size, multiple dilated arterial feeders from anterior and posterior circulation and
\end{abstract}

external carotid arteries, high blood flow, vascular steel from the surrounding brain, enlarged draining veins, profound venous drainage and location in eloquent area. Giant AVMs with high flow nidus, causing a great degree of vascular steel in the surrounding brain, with hypoperfusion of normal parenchyma may develop early normal perfusion pressure breakthrough. Total resection in grade V AVMs can be performed with minimal transient morbidity and favorable outcome. Total resection permits control of intractable seizures with reduced dose of antiepileptic therapy.

Key words: arteriovenous malformation surgery, giant arteriovenous malformation, grade V Spetzler-Martin arteriovenous malformation

\section{Background}

Arteriovenous malformations (AVMs) contain a tangled cluster of vessels, called nidus, in which dysplastic arteries are directly connected through shunts with arterialized veins, with no existence of capillary bed. The nidus is usually compact, 
without intervening brain parenchyma. Dysplastic arteries lack muscularis layer and red draining veins are enlarged and carry oxygenated blood. AVMs are congenital lesions, which occur during the late somite stages of the fourth week of embryonic life.

AVMs were first described by Luschka and Virchow in the mid 1800s, Giordano performed the first surgical exposure of an AVM in 1890, Krause attempted surgical exclusion of an AVM by ligating its feeding arteries in 1908 and Olivecrona performed the first surgical excision of an AVM in 1932. (1)

The incidence of AVMs ranges between 0.15 and $3 \%(12 ; 19)$ and the prevalence is $0.14 \% \quad(1 ; 6)$. AVMs accounts for approximately $6 \%$ of all intracranial lesions and $11 \%$ of all cerebrovascular malformations. (5)

AVMs become clinically manifest in young people, the average age at diagnostic being 33 years, and about $64 \%$ of patients with AVMs are diagnosed before age 40. (5; 6 ; 14) The main clinical forms of presentation are rupture, with consequent hemorrhage and seizures. Other symptoms are neurological deficits secondary to mass effect and intracranial hyperpressure, ischemia of the adjacent brain secondary to vascular steal and hydrocephalus due to venous hypertension.

Treatment of giant AVMs is challenging. According to Spetzler-Martin scale, grade V AVMs have a nidus bigger than $6 \mathrm{~cm}$ in diameter, profound venous drainage and are located in eloquent areas. Surgery is the treatment of choice in AVMs, total resection offering cure of the lesion, eliminating the risk of rupture and improving seizure control. The morbidity and mortality rate in operated grade $\mathrm{V}$ AVMs is high. $(5 ; 7 ; 11 ; 16)$

\section{Case presentation}

We report a case of a giant left frontal AVM, grade V Spetzler-Martin, operated into the Fourth Department of Neurosurgery, Emergency Clinical Hospital Bagdasar-Arseni. We reviewed medical records, imaging, surgical treatment and follow-up.

A 39 years old woman, right-handed was admitted in our department for headache, generalized seizures and postictal right hemiparesis and transient aphasia. The onset of seizures was at age 7 , and they were refractory to antiepileptic drugs. The last antiepileptic scheme contained levetiracetam (Keppra) $1000 \mathrm{mg} / \mathrm{d}$, topiramat (Topamax) $400 \mathrm{mg} / \mathrm{d}$ and valproic acid (Depakine chrono) 1500 $\mathrm{mg} / \mathrm{d}$. Reviewing the medical history, we found out that the patient was previous diagnosed, in another neurosurgical department, with left frontal AVM, grade Spetzler-Martin VI, and therefore was refused for any curative treatment. On admission general physical exam was within normal limits. Neurological exam found mild right hemiparesis and hyperactive deep tendon reflexes in the right hemibody.

Blood analysis $\quad(\mathrm{Hg}=13.6 \mathrm{~g} / \mathrm{dl})$, pulmonary $\mathrm{x}$-ray and electrocardiography showed normal aspect.

Cerebral CT-scan showed giant left frontal AVM, $7 / 6 \mathrm{~cm}$ in diameter, with multiple serpiginous vessels and left parasagittal parietal porencephalic cyst secondary to previous bleeding (Figure 1).

Cerebral MRI showed a giant left frontal AVM, sizing $7 / 6.4 / 5 \mathrm{~cm}$, with a tangle of multiple flow void sinuous vessels, with mass effect on the frontal horn of the left lateral ventricle and enlarged ventricles. Part of the nidus was located into the left motor area. 

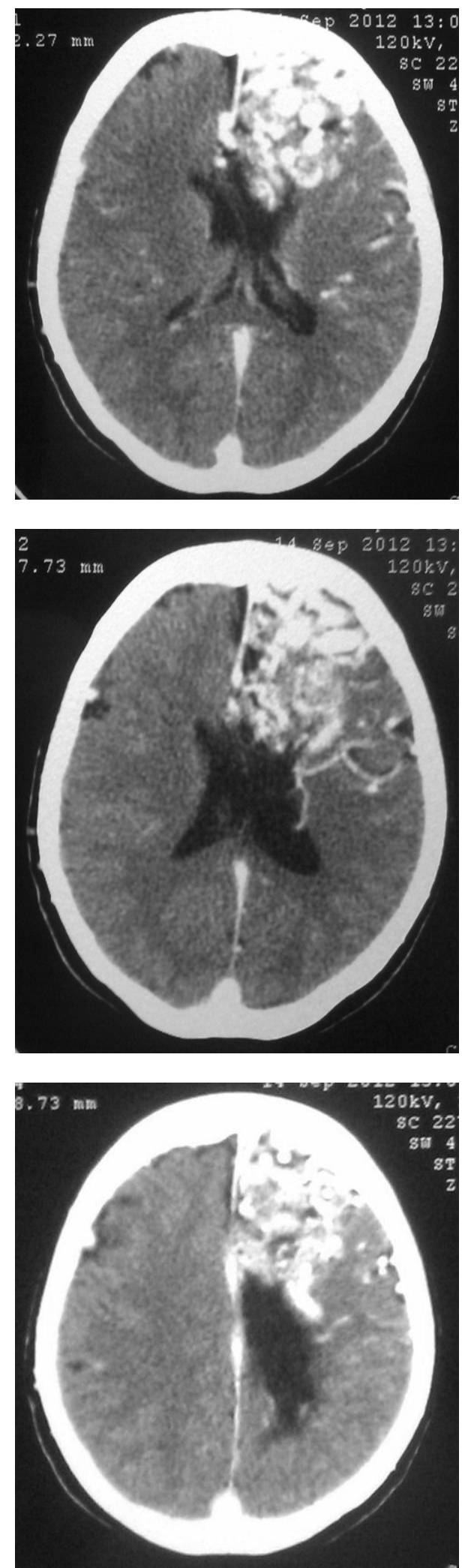

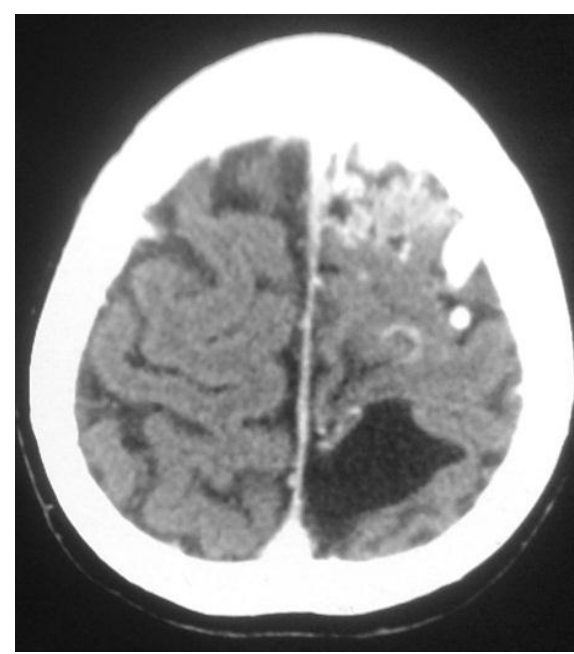

Figure 1 Cerebral CT scan. Giant left frontal AVM, $7 / 6 \mathrm{~cm}$, with mass effect on the frontal horn of the left lateral ventricle and enlarged ventricles, left parasagittal parietal porencephalic cyst

Middle cerebral arteries, anterior cerebral arteries and anterior communicating artery were enlarged due to increased flow. The left internal carotid artery was also enlarged and measured 7 $\mathrm{mm}$ in diameter. Venous sinuses (predominantly sagittal and transverse sinus) were grossly enlarged, the sagittal sinus measuring $24 \mathrm{~mm}$. In the left temporal lobe and left hippocampus there were multiple enlarged sinuous vessels. A porencephalic cavity measuring 41/27 mm was seen left parietal parasagittal.

Cerebral angio-MRI showed complex, giant AVM, containing a large left frontal nidus with feeding arteries from left anterior cerebral artery and anterior communicating artery. Large veins drained into the superior sagittal sinus. The AVM had mass effect, compressing the left lateral ventricle.

Six vessels angiography showed giant left frontal AVM with feeding arteries from left middle cerebral artery, left anterior cerebral artery, anterior communicating artery, right anterior cerebral artery and left external 
carotid artery. Right A1 segment, left A1 segment and left middle cerebral artery were enlarged from increased flow. Midline shift to the right of the cerebral vessels was seen. Venous drainage was superficial into the superior sagittal sinus, through 3 sinuous and ectatic veins, and profound via straight sinus into the inferior sagittal sinus. The AVM was also injected from the vertebrobasilar circulation via left posterior communicating artery and from arterial feeders from left posterior cerebral artery (Figure 2).
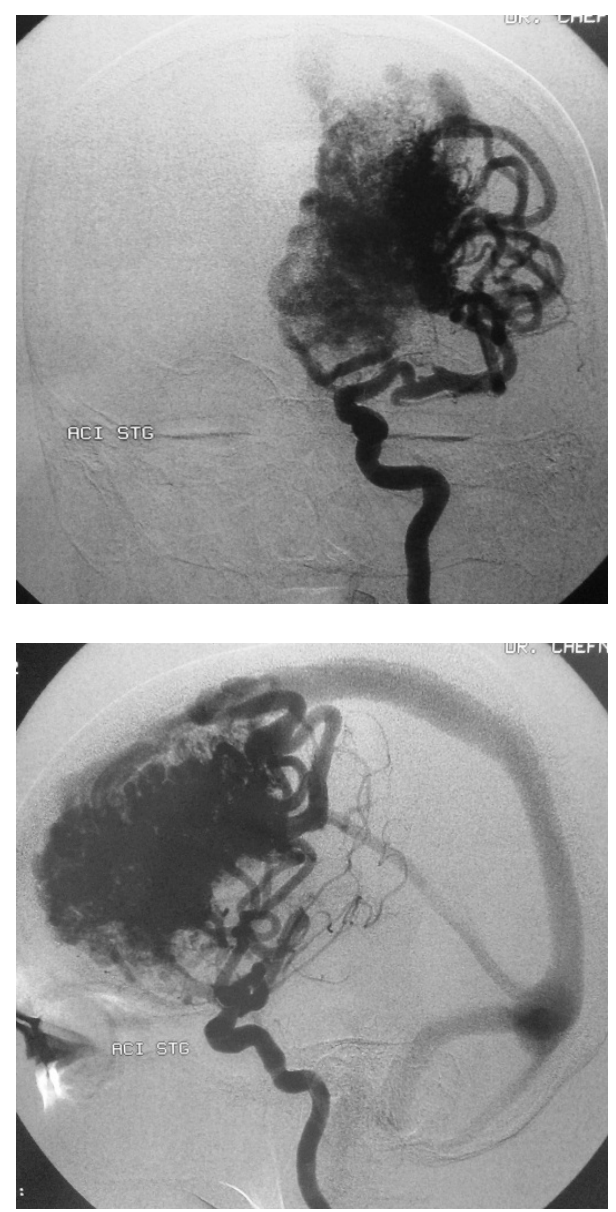
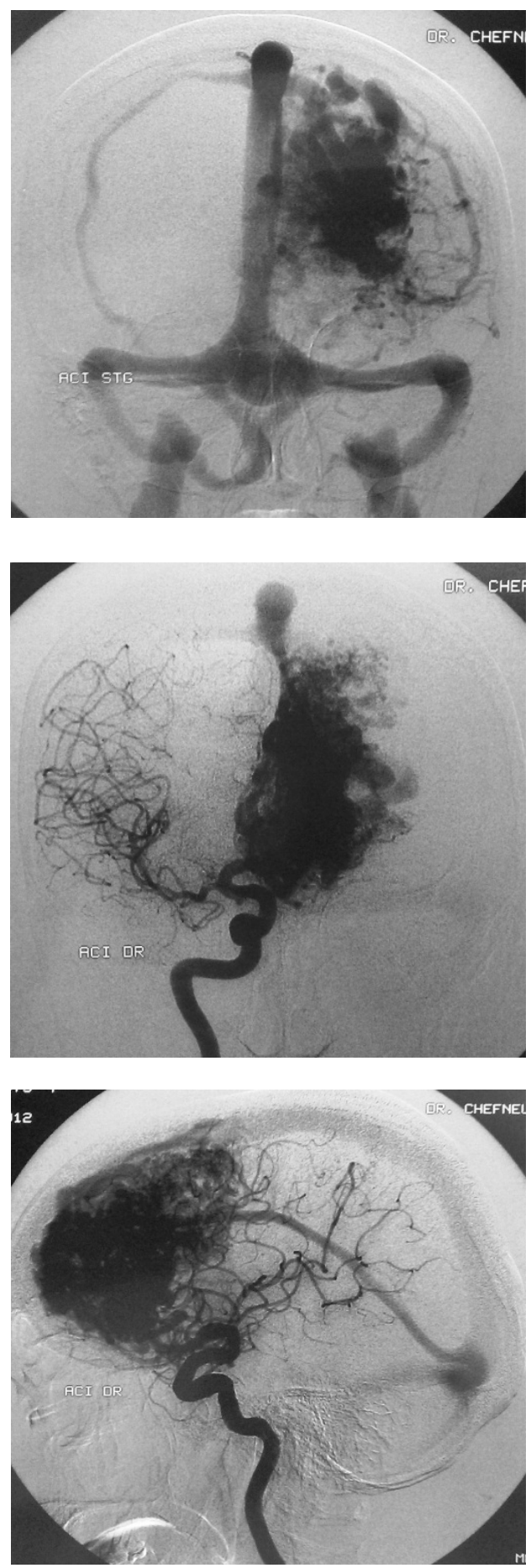
DOI: $10.2478 / \mathrm{v} 10282-012-0020-3$

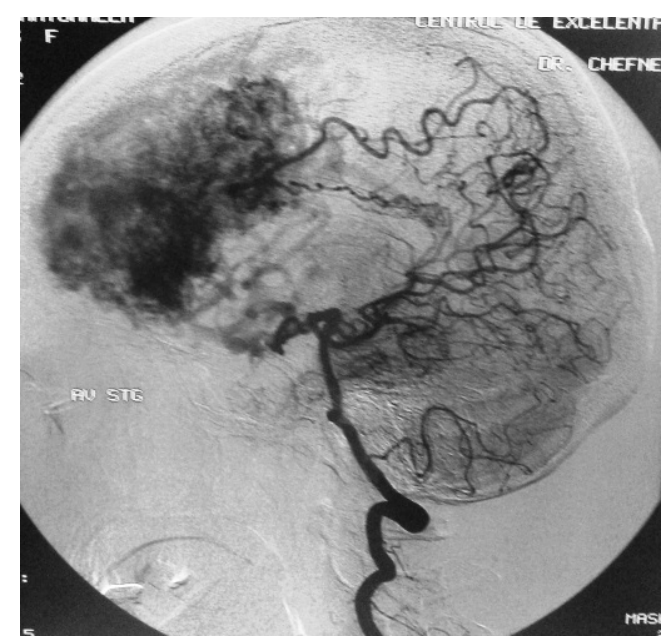

Figure 2 Cerebral angiography. Giant left frontal AVM with feeding arteries from left middle cerebral artery, left anterior cerebral artery, anterior communicating artery, right anterior cerebral artery, left posterior communicating artery and left posterior cerebral artery. Flow-related enlargement of right A1 segment, left A1 segment and left middle cerebral artery. Superficial and profound venous drainage

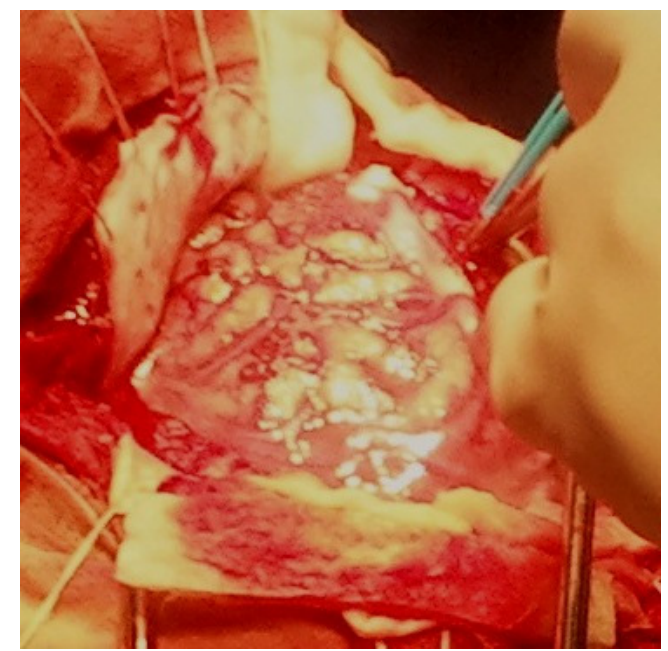

Figure 3 Intraoperatory aspect. Corticalized giant left frontal AVM, with feeding arteries coming from superficial branches from left middle cerebral artery

The patient received Dexamethasone 16 $\mathrm{mg} / \mathrm{d}$, Mannitol 20\% $250 \mathrm{ml} / \mathrm{d}$, Furosemid $40 \mathrm{mg} / \mathrm{d}$, Levetiracetam $1000 \mathrm{mg} / \mathrm{d}$, Topiramat $400 \mathrm{mg} / \mathrm{d}$, Valproic acid 1500 $\mathrm{mg} / \mathrm{d}$, analgesics, intravenous hydration and gastro-protective drugs. Pre-anesthetic consult found a risk score ASA IV.

Patient underwent surgery, under general anesthesia. The patient was placed in supine position and the head was turned $30^{\circ}$ to the right. We performed a large left frontoparietal bone flap; dura mater was cut in a curvilinear fashion. After dural opening, the AVM could be seen on the cortical surface on approximately $20 \mathrm{~cm} 2$. On the cortical surface, the AVM presented multiple feeding arteries from superficial branches of left middle cerebral artery and a large draining vein entering the dura in closed vicinity with superior sagittal sinus (Figure 3). We began to circumferentially dissect the nidus of the AVM, progressively occluding feeding arteries from left middle cerebral artery. Dissection followed the gliotic layer surrounding the AVM. In the postero-superior part of the nidus we indentified and occluded a large feeding artery coming from the left posterior cerebral artery. Dissection progresses towards the midline with occlusion of feeding arteries arising from right internal carotid artery. Feeding arteries were sealed off using electrocoagulation or clipping; a total of 8 Yasargil vascular titanium clips were used. After occluding all feeding arteries and fully circumferentially dissection of the nidus, 2 additional vascular clips were needed to occlude draining veins into the superior and inferior sagittal sinuses. Careful hemostasis was done using electrocoagulation, abundant lavage with saline, $\mathrm{H}_{2} \mathrm{O}_{2}$, haemostatic agents and muscle. The hemostasis posed great difficulties due to brisk profuse bleeding secondary to early normal perfusion pressure breakthrough. Closure and anchoring of dura mater, epidural external draining tube, and the bone flap was replaced back and fixed in place. The scalp 
was closed with simple sutures in anatomical layers.

Operating time was 8 hours. During surgery the patient received 2 whole blood units, 9 fresh frozen plasma units, 16 packed red blood cells and 2 platelet concentrates.

The patient was taken into Intensive Care Unit, and was kept sedated 36 hours. Postoperative outcome was favorable, patient was extubated after 36 hours, regained consciousness, obeyed commands, had no speech disturbances and presented right hemiparesis. The patient presented postoperative acute anemia, $\mathrm{Hg} 6.08 \mathrm{~g} / \mathrm{dl}$, which was corrected using blood transfusions. During next days the neurological outcome was favorable, right hemiparesis improved. Following operation the patient presented no seizure, under antiepileptic drugs.

Control cerebral CT scan showed minimal left frontal bleeding at the resection site, without mass effect (Figure 4). Control 4 vessels cerebral angiography revealed the absence of vascular nidus or abnormal vessels (Figure 5).

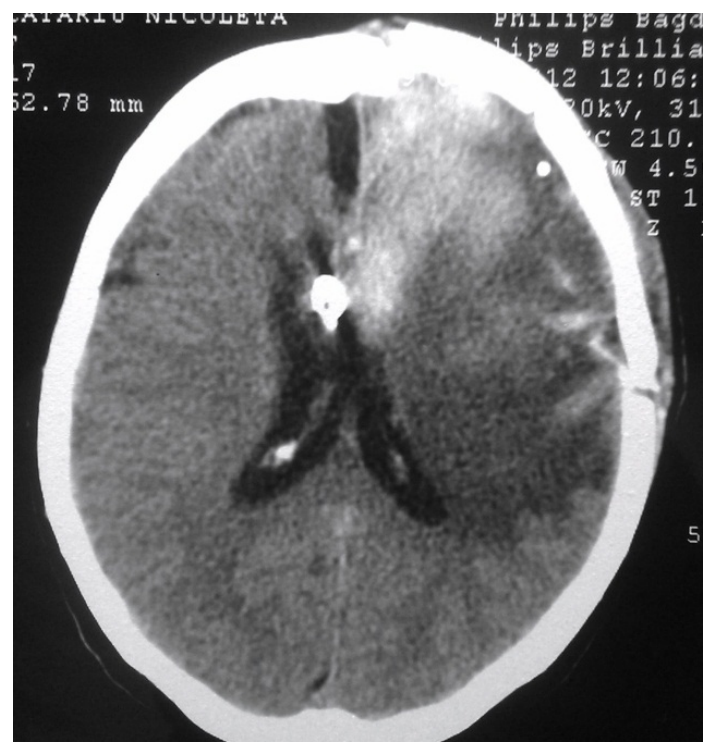

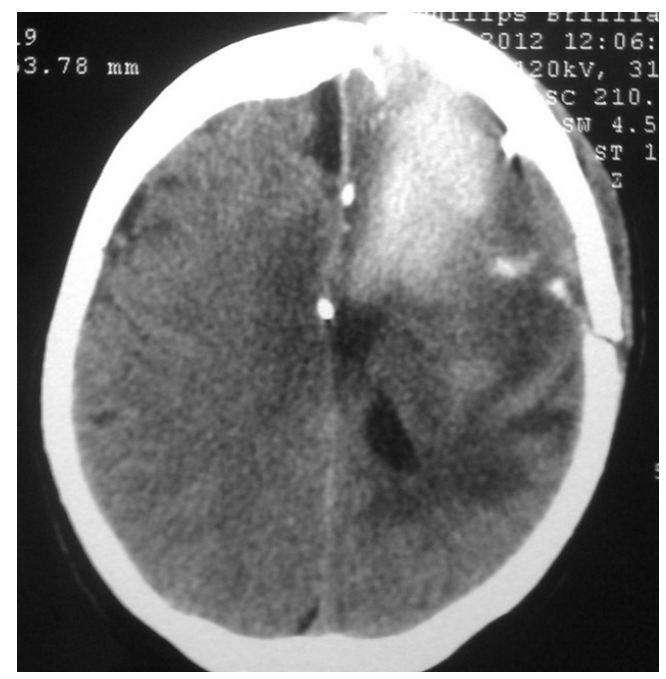

Figure 4 Postoperatory cerebral CT scan. Minimal left frontal bleeding at the resection site, without mass effect
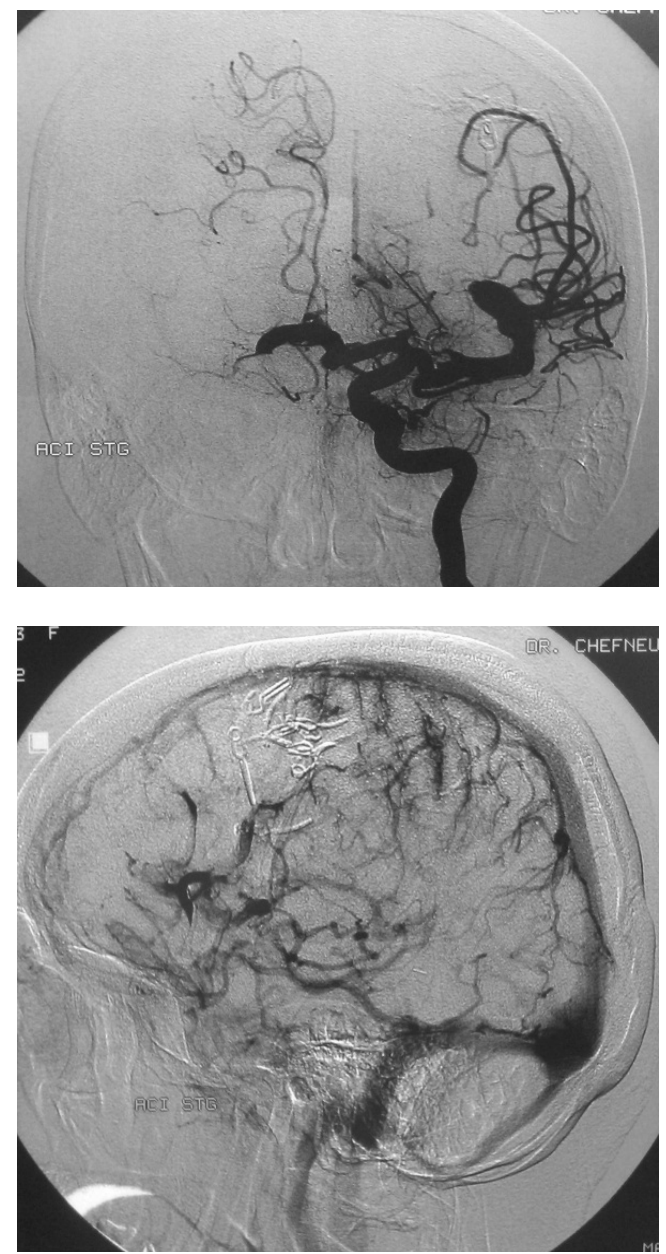
DOI: $10.2478 / \mathrm{v} 10282-012-0020-3$
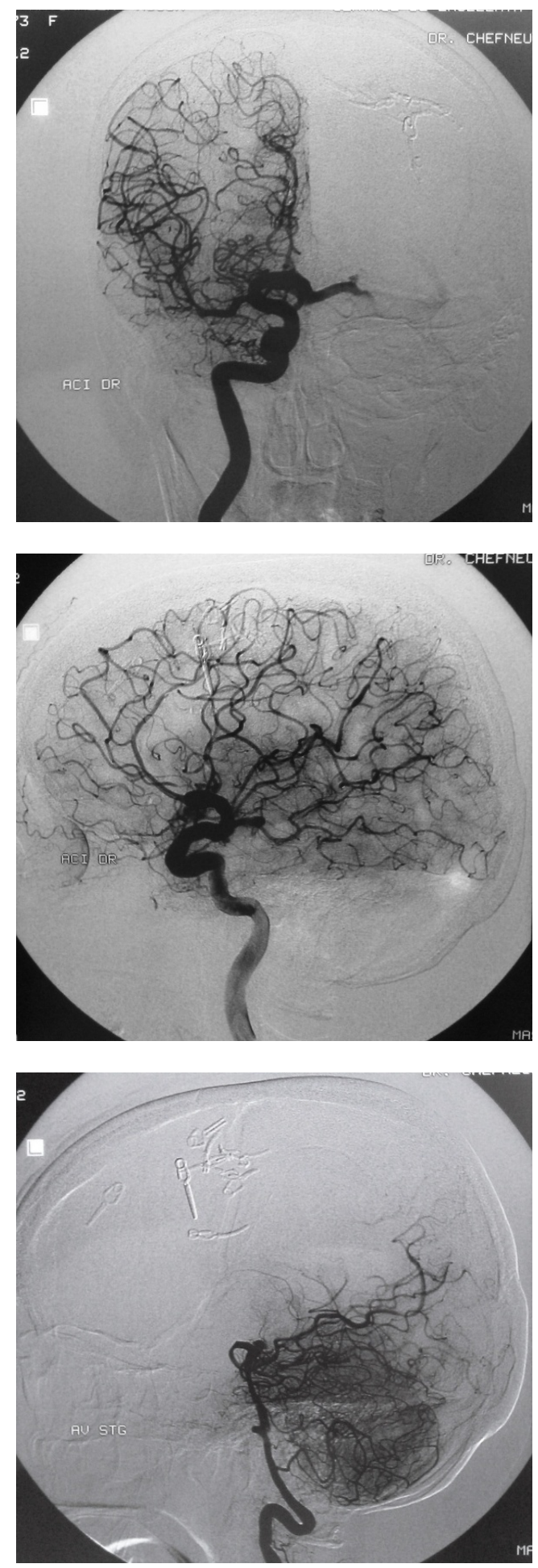

Figure 5 Postoperatory cerebral angiography. Absence of vascular nidus or abnormal vessels
The patient was discharge 26 days after surgery, neurologically improved, presenting only mild right hemiparesis and seizure free. At two month follow-up the patient regained full motor function, she presented no seizures under progressively reduced doses of antiepileptic therapy.

\section{Discussions}

Giant AVMs are lesions with nidus greater than $6 \mathrm{~cm}$ in diameter. Most common forms of clinical presentation of AVMs are hemorrhage or seizures. Our patient had imagistic history of previous bleeding, but intractable seizures were clinically significant. Large AVMs are more likely to present with seizure, because their large size make them more likely to involve the brain tissue. (6)

Persistent, intractable seizures under full dose of antiepileptic drugs severely affect the quality of life. Our patient presented incontrollable seizure for 32 years, in spite of full dose polytherapy anticonvulsivants, that severely impaired her quality of life. Seizures may occur because the surrounding ischemic brain, secondary to vascular steal, has lower thresholds for seizures and higher susceptibility to epileptogenic activity, than normally perfused brain. $(3 ; 18)$

The patient was diagnosed in another department with AVM grade VI SpetzlerMartin. Grade VI AVMs are considered untreatable lesion by any means: surgery, radiosurgery, embolization. If for grades I to $\mathrm{V}$ there are specific criteria and grade of the AVM is easy calculated, by summing points assigned to three criteria (size, eloquence of the brain and pattern of venous drainage) classification as grade VI AVM is subjective and the experience of the surgical team matters more. Unlike grade 
VI, grade $\mathrm{V}$ AVMs may be suitable for surgery. A study regarding large AVMs, grade IV and $\mathrm{V}$, found a risk of hemorrhage of $10.4 \%$ per year in patients with residual nidus, compared with natural history of $1.5 \%$ per year rate of hemorrhage, and the authors concluded that surgery is indicated in such cases only if they are symptomatic and the AVM impairs the quality of life. (7)

Surgery is the treatment of choice in AVMs, being the only one capable of preventing hemorrhage and controlling seizures, but it can only be perform in cases in which the lesion can be removed with acceptable morbidity and mortality risks. The aim of surgery is the complete removal of the nidus. Surgical risk, concerning major neurological deficits and death, is directly proportionate with Spetzler-Martin grade of AVMs. (16) Grade V SpetzlerMartin poses major surgical risks, due to large nidus measuring more than $6 \mathrm{~cm}$ in diameter, deep venous drainage and extension of a part of the nidus into motor area. Besides this grading system a supplementary grading scale was propose in order to predict more accurate the outcome after surgery, that included also age, diffuseness of the nidus and hemorrhagic presentation. (10) Taken into consideration this supplementary scale, our patients had a diffuse nidus and previous bleeding, seen as a parietal parasagittal porencephalic cavity. Once surgery is chosen, one must considerate also arterial supply. Giant AVMs presented complex arterial feeders arising from multiple main brain arteries, which enter the nidus in various sites. Careful inspection of angiographic images helps to indentify all feeding arteries and draining veins. Our patients had arterial feeders coming from left middle cerebral artery, left anterior cerebral artery, anterior communicating artery, right anterior cerebral artery, left posterior cerebral artery, left posterior communicating artery and left external carotid artery. Angiography is also useful to reveal passage arteries, that only pass or skirt the nidus and pass through to supply normal brain and associated flowrelated aneurysm. We did not identify such arteries or flow related aneurysms. Other difficulties posed by surgery are related to location of the AVM in the dominant hemisphere and extension of the nidus to the motor area.

Surgery for AVMs must take into consideration several aspects. A large bone flap is needed, that fully circumscribes the nidus and offer access to vascular supply. Bone flap must be larger than that performed for same size tumors, because unlike tumors AVMs cannot be debulked from inside, the nidus cannot be retracted and draining veins may restrict access. (9) If vascular supply from the left external carotid artery is present, the surgeon must expect an important bleeding during opening of the skull. Dura mater must not be torn during bone flap removal, and cutting it must be done with great care, because draining veins can travel within the dura mater, and these veins must be preserved until the end of the operation. We found a draining vein that was entering the dura mater near the superior sagittal sinus. Inspection of the brain revealed a corticalized AVM and identified multiple arterial feeders and a superficial draining vein. When operating an AVM, the surgeon must keep in mind that it is triangular in shape with the base towards the cortex and the apex towards the ventricle. As a golden rule, feeding arteries must be sealed off first and veins must be kept patent till the end, to prevent engorgement of the nidus and 
rupture. $(4 ; 6 ; 9 ; 11)$ We begun devascularization of the AVM, we occluded first cortical feeders from superficial branches of left middle cerebral artery, than we started dissection by circumscribing the nidus. Dissection progressed in the surrounding gliotic layer, further sealing off feeding arteries from left middle cerebral artery. Dissection of the posterior part of the nidus was facilitated by the presence of a porencephalic cavity secondary to previous bleeding. The postero-superior part of the nidus was fed by a large artery coming from the left posterior cerebral artery. Vascular supply of the medial part of the nidus came from branches from right internal carotid artery. The deep part of the nidus, which came up to the frontal horn of the lateral ventricle, was most challenging to dissect because here there were small high-pressure feeders arising from chroidal and ependymal arteries and fragile small veins, that are difficult to control and can retract into adjacent parenchyma, plus when reaching the deep part of the nidus occurs a vigorous attempt of recanalization the AVM. $(9 ; 11)$ The tip of the AVM reaches the left lateral ventricle, therefore the surgeon must be prepared to enter into the ventricle. At the end of the operation draining veins were occluded. A total number of 10 Yasargil vascular clips were used, 8 for sealing the arteries and 2 for veins. Vascular clips were preferred instead of coagulation of the vessel, because of their big diameter, which made coagulation alone impossible or ineffective.

A possible intraoperative difficulty may be premature occlusion of a major draining vein, which leads to nidus rupture and massive bleeding. This is the reason why before occluding a vessel, a temporary occlusion must be performed, and nidus changes must be observed. If the vessel is a vein the nidus will become tensed. In this case the temporary clip must be removed immediately and the vein must be preserved until the end of the operation. If the vessel is an artery, the nidus will not change turgor or it may shrink. Care must be taken not to mistake a vein for an artery, because veins are red, containing oxygenated blood and enlarged.

Excessive intraoperative bleeding required massive blood and blood products transfusions. Massive transfusions impair coagulation (15), making hemostasis more difficult. Besides coagulation impairment, massive transfusions lead to acidosis, hypothermia, acid-base imbalances, electrolyte abnormalities (hypocalcemia, hypomagnesemia, hypokalemia, hyperkalemia), citrate toxicity and transfusion-associated acute lung injury. (15) Even with all massive transfusions the patient presented acute postoperative anemia, which was aggressively corrected, to improve oxygen delivery to the brain tissue.

Normal perfusion pressure breakthrough occurs due to loss of autoregulation of cerebral vessels. $(6 ; 17) \mathrm{A}$ large amount of blood that was flowing through the nidus is redistributed to the adjacent brain after AVM resection. Vessels supplying normal brain present long-term vasodilatation due to prolong ischemia. In time, they lose the capacity of vasoregulation and when flow is redistribute these vessels, they are incapable of vasoconstriction, leading to brain swelling and hemorrhage. At the end of the operation an early normal perfusion pressure breakthrough occurred, that required prolong and careful hemostasis. This is due to large size of the AVM, which 
was stealing a large amount of blood, fact seen on cerebral angiography. Right A1 segment, left A1 segment and left middle cerebral artery were enlarged from increased flow, and arteries supplying normal brain are barely visible, suggesting long-term ischemia. The presence of regional hypoperfusion due to vascular steal indicates a higher risk for normal perfusion pressure breakthrough occurrence. Risk of developing normal perfusion pressure breakthrough is higher in large AVMs, with high flow, steal from vertebrobasilar system or controlateral circulation and feeders from external carotid artery. (11)

The patient presented right hemiparesis, intensive kinesiotherapy program was initiated and motor deficit slowly improved, at discharge she presented mild to moderate right hemiparesis, predominantly brachial and on two month follow-up she regained full motor function. Total resection of the AVM permitted full seizures control with reduced dose of antiepileptic therapy.

In giant AVMs multimodal treatment can also be applied. Some authors recommend staged stereotactic surgery (4; $8)$, stereotactic radiation $(2 ; 20)$ or embolization $(11 ; 13 ; 21)$ in giant AVMs to convert them to surgery.

\section{Conclusions}

Surgery is the therapy of choice in AVMs, because it is the only curative treatment, capable of preventing hemorrhage and controlling seizures. Management in grade $\mathrm{V}$ AVMs is challenging, because of their large size, multiple dilated arterial feeders from anterior and posterior circulation and external carotid arteries, high blood flow, vascular steel with prolong hypoperfusion of the surrounding brain, grossly enlarged draining veins, profound venous drainage and location in eloquent area. Giant AVMs with high flow nidus, causing a great degree of vascular steel in the surrounding brain, with hypoperfusion of normal parenchyma may develop early normal perfusion pressure breakthrough. Total resection in grade V AVMs can be performed with minimal transient morbidity and favorable outcome. Total resection of the AVM permitted control of intractable seizures with reduced dose of antiepileptic therapy.

\section{Correspondence:}

Aurelia Mihaela Sandu, address: Emergency

Clinical Hospital Bagdasar-Arseni, No. 10-12,

Berceni Street, Sector 4, Bucharest; e-mail: aurasandu@gmail.com; tel.0724.263.023

\section{References}

1. Altschul D, Smith ML, Sinson GP. (2011). Intracranial arteriovenous malformation. Medscape eMedicine[Surgery/Neurosurgery/Vascular].

2. Andisheh B, Brahme A, Bitaraf MA, Mavroidis P, Lind BK. (2009). Clinical and radiobiological advantages of single-dose stereotactic light-ion radiation therapy for large intracranial arteriovenous malformations. Technical note. J Neurosurg 111, 919926.

3. Congar P, Gaiarsa JL, Popovici T, Ben-Ari Y, Crepel V. (2000). Permanent reduction of seizure threshold in post-ischemic CA3 pyramidal neurons. J Neurophysiol 83, 2040-2046.

4. Deruty R, Turjman F, Pelissou-Guyotat I. (2006). Surgical managemnt of cerebral arteriovenous malformations. In Schmidek HH, Roberts DW (eds), Operative neurosurgical techniques - indications, methods, and results. Philadelphia, Saunders Elsevier, pp 1263-1277.

5. Gorgan RM: Malformațiile arterio-venoase cerebrale. (2007). In Popescu I (ed), Tratat de chirurgie. București, Ed. Academiei Române, 2007, pp 323-335.

6. Greenberg MS. (2010). Vascular malformations. In Greenberg MS (ed), Handbook of neurosurgery. New York, Thieme Medical Publisher, pp 1098-1142.

7. Han PP, Ponce FA, Spetzler RF. (2003). Intention to treat analysis of Spetzler-Martin grade IV and V AVMs: natural history and treatment paradigm. J Neurosurg 
DOI: $10.2478 / \mathrm{v} 10282-012-0020-3$

98, 3-7.

8. Hanakita S, Koga T, Shin M, Shojima M, Igaki H, Saito N. (2012). Role of stereotactic radiosurgery in the treatment of high-grade cerebral arteriovenous malformation. Neurol Med Chir (Tokyo) 52, 845-851.

9. Komotar RJ, Strake RM, Otten ML. (2010). Surgical treatment of cortical arteriovenous malformations. In Connolly Jr. ES, McKhann II GM, Huang J, Choudhri TF, Komotar RJ, Mocco J (eds), Fundamentals of operative techniques in neurosurgery. New York, Thieme Medical Publishers, pp 138-143.

10. Lawton MT, Kim H, McCulloch CE, Mikhak B, Young WL. (2010). A supplementary grading scale for selected patients with brain artriovenous malformations for surgery. Neurosurgery 66, 702-713.

11. Marciano FF, Vishteh AG, Apostolides PJ, Spetzler RF. (2000). Arteriovenous malformations supratentorial. In Kaye AH, Black P (eds), Operative neurosurgery. London, Harcourt Publishers Limited, pp 1079-1091.

12. Misra M, Aletich V, Charbel FT, Debrun GM, Ausman JI. (2000). Multidisciplinary approach to arteriovenous malformations. In Kaye AH, Black PM (eds), Operative neurosurgery. London, Harcourt Publishers Limited, pp 1137-1151.

13. Natarajan SK, Ghodke B, Britz GW, Born DE, Sekhar LN. (2008). Multimodality treatment of brain arteriovenous malformations with microsurgery after embolization with onyx: single-center experience and technical nuances. Neurosurgery 62, 1213-1225.

14. Ondra SL, Troupp H, George ED, Scawab K.
(1990). The natural history of symptomatic arteriovenous malformations of the brain: a 24-year follow-up assessment. J Neurosurg 73, 387-391.

15. Sihler KC, Napolitano LM. (2010). Complications of massive transfusion. Chest 137, 209-220.

16. Spetzler RF, Martin NA. (1986). A proposed grading system for arteriovenous malformations. J Neurosurg 65, 476-483.

17. Spetzler RF, Wilson CB, Weinstein P, Mehdorn M, Townsend J, Telles D. (1978). Normal perfusion pressure breakthrough theory. Clin Neurosurg 25, 651672.

18. Srejic LR, Valiante TA, Aarts MM, Hutchinson WD. (2013). High-frequency cortical activity associated with postischemic epileptiform discharges in an in vivo rat focal stroke model. J Neurosurg published before print [published online February 15, 2013; DOI: 10.3171/2013.1.JNS121059.].

19. Stapf C, Mast H, Sciacca RR, Berenstein A, Nelson PK, Gobin YP, Pile-Spellman J, Mohr JP. (2003). The New York islands AVM study: design, study progress, and initial results. Stroke 34, e29-34.

20. Xiao F, Gorgulho AA, Lin CS, Chen CH, Agazaryan N, Vinuela F, Selch MT, De Salles AA. (2010). Treatment of giant cerebral arteriovenous malformation: hypofractionated stereotactic radiation as the first stage. Neurosurgery 67, 1253-1259.

21. Xu F, Ni W, Liao Y, Gu Y, Xu B, Leng B, Song D. (2011). Onyx embolization for the treatment of brain arteriovenous malformations. Acta Neurochir (Wien) 153, 869-878. 